\title{
Synthesis, Characterization and Biological Activities of Novel (E)-3-(1-(Alkyloxyamino)ethylidene)-1-alkylpyrrolidine-2,4-dione Derivatives
}

\author{
Zhao-Yong Zhu, ${ }^{\dagger, *}$ Qing-Ming Shi, ${ }^{\dagger, \downarrow}$ Bao-Feng Han, ${ }^{\dagger,+}$ Xian-Feng Wang, ${ }^{\dagger, ~ S h e n g ~ Q i a n g, ~}{ }^{\S}$ and Chun-Long Yang ${ }^{\dagger, * *}$ \\ ${ }^{\dagger}$ Department of Chemistry, College of Science, Nanjing Agricultural University, Nanjing 210095, P. R. China \\ *E-mail: chunlongyang@yahoo.com.cn \\ ${ }^{\sharp}$ Jiangsu Key Laboratory of Pesticide Science, Nanjing Agricultural University, Nanjing 210095, P. R. China \\ ${ }^{\S}$ College of Life Science, Nanjing Agricultural University, Nanjing 210095, P. R. China \\ Received May 24, 2010, Accepted July 4, 2010
}

\begin{abstract}
Twenty novel tetramic acid derivatives (E)-3-(1-(alkyloxyamino)ethylidene)-1-alkylpyrrolidine-2,4-diones were synthesized by the reaction of 3-(1-hydroxyethylidene)pyrrolidine-2,4-diones with $O$-alkyl hydroxylamines. The title compounds were confirmed by IR, ${ }^{1} \mathrm{H}$ NMR, MS and elemental analysis. The structure of compound 6 r was further verified by X-ray diffraction crystallography. The bioassays showed that most of the title compounds exhibited noticeable herbicidal and fungicidal activities.
\end{abstract}

Key Words: Pyrrolidine-2,4-dione, Oxime ether, Synthesis, Crystal structure, Biological activity

\section{Introduction}

To study the active material of natural products such as nicotine, toosendanin, pyrethrum, and essential oil of Caesulia axillaris etc., ${ }^{1-4}$ is very common in the field of medicinal chemistry. Pyrrolidine-2,4-dione (also known as tetramic acid) is one of the outstanding kinds of natural compounds, some naturally bioactive tetramic acids could be seen in Scheme $1 .^{5-8}$ Thereinto, tenuazonic acid (or TeA for short), a metabolic toxin from widely differing phytopathogenic fungi, has been found possessing herbicidal, antibacterial, and antitumor activities, ${ }^{9-11}$ it has evoked many chemist's great interest. Up to now, multifarious tenuazonic acid derivatives have been synthesized to screen out new compounds with biological activity. For example, a series of 3( $\alpha$-hydroxy-benzylidene)pyrrolidine-2,4-dione derivatives were reported displaying high herbicidal activity, ${ }^{12}$ another kind of tenuazonic acid derivatives with oxime ether moiety were presented showing obvious herbicidal and antifungal activities simultaneously. ${ }^{13}$

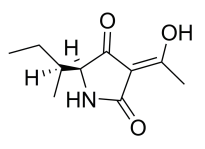

Tenuazonic acid

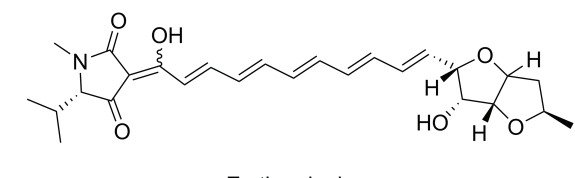

Erythroskyrine
As an important kind of pesticides the oxime ether derivatives have been applied as insecticides, fungicides, and herbicides. Sulfoxime is a new insecticide with a structure of propiophenone oxime ether, having the characteristics of high activity, low toxicity, and low residue. ${ }^{14}$ Cymoxan is the first oxime ether fungicide commercialized by DuPont. ${ }^{15}$ And the first cyclohexanedione herbicide, alloxydim-sodium, with a oxime ether group, is a postemergence herbicide providing excellent control of a wide range of grass weeds. ${ }^{16}$ In this article, alkyl and cycloalkyl were introduced to the 1-position of the heterocycle pyrrolidine-2,4-dione respectively, instead of substituted phenyl or hydrogen reported by Zhu, ${ }^{13}$ to design and synthesize a series of novel tetramic acid derivatives with oxime ether moiety at 3-position. Moreover, these synthesized title compounds were tested to evaluate their herbicidal and fungicidal activities.

\section{Results and Discussion}

Synthesis. The title compounds 6a-t were synthesized by the
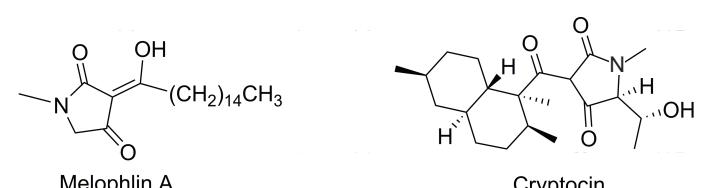

Cryptocin

Scheme 1. Some bioactive naturally existing tetramic acids

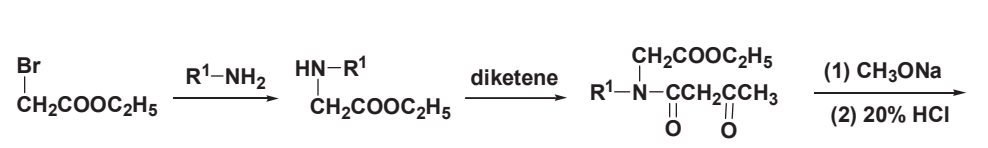

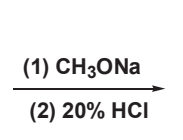

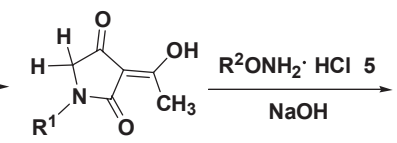

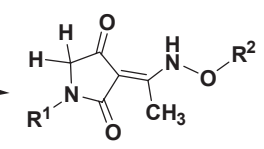

6a-t

$\mathrm{R}^{1}: \mathbf{6 a}-\mathbf{e}=i-\mathrm{C}_{3} \mathrm{H}_{7}, 6 \mathbf{f - j}=n-\mathrm{C}_{4} \mathrm{H}_{9}, \mathbf{6 k}-\mathbf{o}=t-\mathrm{C}_{4} \mathrm{H}_{9}, \mathbf{6 p}-\mathbf{t}=$ cyclo $-\mathrm{C}_{6} \mathrm{H}_{11}$

$\mathrm{R}^{2}: 6 \mathbf{a}, 6 \mathbf{6 f}, 6 \mathbf{k}, 6 \mathbf{p}=n-\mathrm{C}_{3} \mathrm{H}_{7}, 6 \mathbf{b}, 6 \mathbf{g}, 6 \mathbf{l}, 6 \mathbf{q}=i-\mathrm{C}_{3} \mathrm{H}_{7}, 6 \mathbf{c}, 6 \mathbf{h}, 6 \mathbf{m}, 6 \mathbf{r}=\mathrm{CH}_{2}-\mathrm{CH}=\mathrm{CH}_{2}, 6 \mathbf{d}, 6 \mathbf{i}, 6 \mathbf{n}, 6 \mathbf{s}=n-\mathrm{C}_{4} \mathrm{H}_{9}, 6 \mathbf{e}, 6 \mathbf{j}, 6 \mathbf{0}, 6 \mathbf{t}=\mathrm{CH}_{2} \mathrm{C}_{6} \mathrm{H}_{5}$.

Scheme 2. The synthetic route and the structures of title compounds 
reaction of 1-alkyl-3-(1-hydroxyethylidene)pyrrolidine-2,4diones 4 with $O$-alkyl hydroxylamines 5 in the presence of sodium hydroxide. The detailed synthetic route and the structures of title compounds are shown in Scheme 2.

Intermediates $\mathbf{4}$ were prepared according to the method including three steps. Firstly, ethyl bromoacetate was reacted with amine in ether to obtain ethyl 2-(alkylamino)acetate 2 . Then the treatment of compound $\mathbf{2}$ with diketene under low temperature formed ethyl 2-( $N$-alkyl-3-oxo-butyramido)acetate 3, which was cyclized in the presence of sodium methoxide and acidified with hydrochloric acid to obtain intermediates $4 .{ }^{17}$ The intermediates $5 O$-propyl, $O$-isopropyl, $O$-allyl, $O$-butyl, and $O$ benzyl hydroxylamine hydrochlorides were synthesized with starting materials ethyl acetate and hydroxylamine hydrochloride via a facile three-step procedure including acetylamination, etherification and hydrolyzation. ${ }^{18,19}$

Structure. In the IR spectra of the title compound $\mathbf{6}$ there were relatively strong absorption bands for the carbonyls at around $1680 \mathrm{~cm}^{-1}$ and $1635 \mathrm{~cm}^{-1}$ respectively. The stretching vibration absorption peak of N-H group existed at $3100-3182 \mathrm{~cm}^{-1}$. In the ${ }^{1} \mathrm{H}$ NMR spectra of $6 \mathbf{a}-\mathbf{t}$, the singlet at $\delta_{\mathrm{H}} 3.57-3.76$ assigned to the $\mathrm{C}-\mathrm{H}$ proton of $\mathrm{NCH}_{2}$ and singlet at $\delta_{\mathrm{H}} 2.40-2.54$ assigned to the $\mathrm{C}-\mathrm{H}$ protons of $\mathrm{CH}_{3} \mathrm{C}=\mathrm{N}$. Furthermore, the MS spectra of all the compounds 6 showed the molecular ion peak $\left(\mathrm{M}^{+}, 12\right.$ $100 \%$ ), and other fragmentation ions were consistent with their structures and could be clearly assigned. The data of measured elemental analyses were also consistent with the corresponding calculated ones.

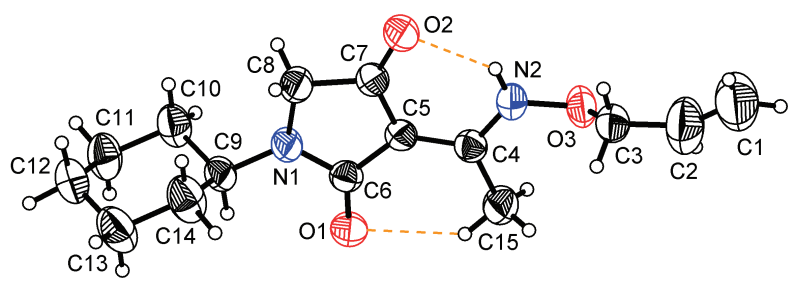

Figure 1. Molecular structure of compound $\mathbf{6 r}$.
In the crystal structure of compound $\mathbf{6 r}$ (Figure 1), the bond $\mathrm{C}(4)-\mathrm{C}(5)$ [1.395(4) $\AA$ ], which was close to $\mathrm{C}=\mathrm{C}$ double bond $[1.38 \AA]$, exhibited $\mathrm{C}=\mathrm{C}$ double bond' property. The bond lengths C(4)-N(2) and C(6)-N(1) were [1.319(3) $\AA$ ] and [1.367(3) $\AA$ ] which were shorter than the normal $\mathrm{C}-\mathrm{N}$ bond distance $[1.47-$ $1.50 \AA]$. The bond lengths $\mathrm{C}(5)-\mathrm{C}(7)$ and $\mathrm{C}(5)-\mathrm{C}(6)$ were $[1.411$ (4) $\AA]$ and $[1.466(4) \AA]$, they were shorter than C-C single bond distance $[1.54 \AA]$. The bonds $\mathrm{C}(6)-\mathrm{O}(1)$ and $\mathrm{C}(7)-\mathrm{O}(2)[1.227$ (4) $\AA$ and 1.236(4) $\AA$, respectively], approaching to the normal $\mathrm{C}=\mathrm{O}$ double bond distance $[1.19-1.23 \AA]$, displayed the property of $\mathrm{C}=\mathrm{O}$ double bond. As a result, there was a electron delocalization between $\mathrm{C}(4)-\mathrm{C}(5)-\mathrm{C}(6)-\mathrm{N}(1)$ and $\mathrm{C}(7)$, and a conjugated system was accordingly formed with $\mathrm{C}(6)=\mathrm{O}(1)$, $\mathrm{C}(7)=\mathrm{O}(2)$.

It is noteworthy that there existed intramolecular $\mathrm{H}$-bondings between $\mathrm{N}(2)-\mathrm{H}(2 \mathrm{~A}) \cdots \mathrm{O}(2)$ and $\mathrm{C}(15)-\mathrm{H}(15 \mathrm{~B}) \cdots \mathrm{O}(1)$ to keep the balance of the molecular structure. In the packing diagram of compound $\mathbf{6 r}$ (Figure 2), the molecules were connected each other through intermolecular $\mathrm{H}$-bondings among $\mathrm{N}(2)-\mathrm{H}(2 \mathrm{~A}) \cdots$ $\mathrm{O}(2), \mathrm{C}(3)-\mathrm{H}(3 \mathrm{~A}) \cdots \mathrm{O}(1)$, and $\mathrm{C}(3)-\mathrm{H}(3 \mathrm{~B}) \cdots \mathrm{O}(2)$ to form a sawtoothed configuration. What's more, the molecular structure of compound $6 \mathbf{r}$ showed $E$ configuration of the ethylidene group and the dione structure of pyrrolidine. The crystal and refinement details of compound 6 r could be seen in Table 1 .

The compounds containing the group of tetramic acid usually tautomerize their structures between ketone form and enol form. ${ }^{20}$ The spectroscopic analysis especially X-ray diffraction crystallography revealed that the title compounds' structure belonged under ketone form. On contray to this, 3-(1-(alkyloxyimino)ethyl)-5-arylidene-4-hydroxypyrroline-2-one derivatives, another oxime ether of tetramic acid, exist in enol form. ${ }^{21}$ By our recent research, it was found that there might be crucial relationship between tautomers and their biological activities, the title compounds exhibited noticeable herbicidal and fungicidal activities simultaneity, but above mentioned 4hydroxypyrroline-2-one derivatives showed only fungicidal activity. ${ }^{21}$ This deduction provided an important guidance for further studies on structural modification of tetramic acid derivatives.

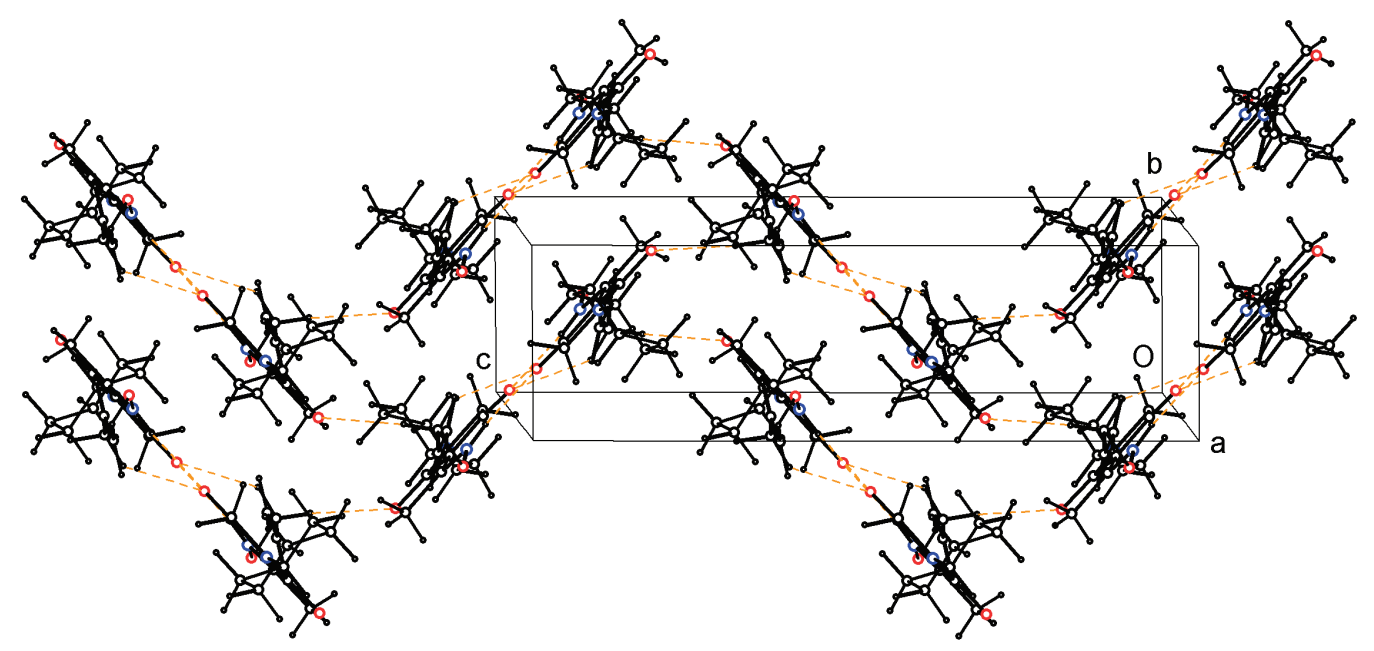

Figure 2. Packing diagram of compound $\mathbf{6 r}$ showing the intermolecular interactions. 
Table 1. Crystal data and refinement details for compound $\mathbf{6 r}$

\begin{tabular}{|c|c|}
\hline Empirical formula & $\mathrm{C}_{15} \mathrm{H}_{22} \mathrm{~N}_{2} \mathrm{O}_{3}$ \\
\hline Formula weight & 278.350 \\
\hline Wavelength $(\AA)$ & 0.71073 \\
\hline Temperature (K) & $296(2)$ \\
\hline Color, Shape & Colorless, Block \\
\hline Crystal system & Monoclinic \\
\hline space group & $C 2 / c$ \\
\hline$a(\AA)$ & $32.778(9)$ \\
\hline$b(\AA)$ & $5.1948(14)$ \\
\hline$c(\AA)$ & $18.025(5)$ \\
\hline$\alpha\left(^{\circ}\right)$ & 90.000 \\
\hline$\beta\left(^{0}\right)$ & $102.734(3)$ \\
\hline$\gamma\left({ }^{0}\right)$ & 90.000 \\
\hline$V\left(\AA^{3}\right)$ & $2993.7(14)$ \\
\hline$Z$ & 8 \\
\hline$D_{\text {calc }}\left(\mathrm{g} \mathrm{cm}^{-3}\right)$ & 1.235 \\
\hline$F(000)$ & 1200 \\
\hline$\mu\left(\mathrm{mm}^{-1}\right)$ & 0.086 \\
\hline Crystal size (mm) & $0.30 \times 0.26 \times 0.24$ \\
\hline$\theta$ Range $\left({ }^{\circ}\right)$ & $2.32-26.00$ \\
\hline Reflection collected & 10958 \\
\hline Independent reflections & 2944 \\
\hline Data/restraints/parameters & $2944 / 1 / 182$ \\
\hline Goodness-of-fit on $F^{2}$ & 1.000 \\
\hline$R$ indices (all data) & $R_{1}=0.0822, w R_{2}=0.1613$ \\
\hline Final $R$ indices $[I>2 \sigma(I)]$ & $R_{1}=0.0678, w R_{2}=0.1565$ \\
\hline
\end{tabular}

\section{Experimental}

The melting points of the products were determined on a WRS-1B digital melting-point apparatus and were not corrected. IR was recorded on a Bruker Tensor 27 FT-IR spectrometer with $\mathrm{KBr}$ disk. Elemental analyses were performed on a Elementar Vario-III CHN analyzer. Mass spectra was recorded on a GC/ MS-QP2010 spectrometer using direct injection technique. ${ }^{1} \mathrm{H}$ NMR spectra was taken on a Mercury plus varian-300 spectrometer with TMS as the internal reference and $\mathrm{CDCl}_{3}$ as the solvent. X-ray diffraction was performed with a Brucker Smart APEX II CCD diffractometer. All reagents were analytical reagent grade or were chemically pure. The solvents were dried prior to use as needed.

General procedure for the synthesis of 1-alkyl-3-(1-hydroxyethylidene)pymolidine-2,4-diones 4 . The solution of amine $(0.3 \mathrm{~mol})$ in ether $(50 \mathrm{~mL})$ was cooled to $0{ }^{\circ} \mathrm{C}$ and ethyl bromoacetate was added gently in $2 \mathrm{~h}$. Then the reaction mixture was stirred for $12 \mathrm{~h}$ at room temperature. After filtering and washing with ether, the filtrate was gradually removed by evaporation under vacuum to give the liquid product ethyl 2-(alkylamino)acetate.

Whereafter, ethyl 2-(alkylamino) acetate $(0.12 \mathrm{~mol})$ was dissolved in benzene $(50 \mathrm{~mL})$, cooled to $10{ }^{\circ} \mathrm{C}$, added diketene $(0.12 \mathrm{~mol})$ slowly in $1 \mathrm{~h}$ and stirred for $10 \mathrm{~h}$ at room temperature. Then the solution of sodium methoxide $(0.12 \mathrm{~mol})$ in $40 \mathrm{~mL}$ methanol was added and refluxed for $4 \mathrm{~h}$. By evaporation under vacuum the solvent was removed and $100 \mathrm{~mL}$ water was added, the impurities were extracted with ether $(25 \mathrm{~mL} \times 3)$ for three times, the aqueous layer was acidified to $\mathrm{pH}=2-3$ with $20 \%$ hydrochloric acid to obtain white solid, which was precipitated, filtered off, dried, recrystallized with ethyl acetate to get intermediates 4 in yield $43.2 \%-62.1 \%$.

3-(1-Hydroxyethylidene)-1-isopropylpymolidine-2,4-dione (4a): white solid; mp $75.8-76.7^{\circ} \mathrm{C}$; yield, $52.0 \%$; IR $\left(\mathrm{KBr}, \mathrm{cm}^{-1}\right)$ v 2977, 1716, 1636, 1468, 1381, 1247, 1229, 976; ${ }^{1} \mathrm{H}$ NMR $\left(\mathrm{CDCl}_{3}, 300 \mathrm{MHz}\right) \delta 1.20\left(\mathrm{~d}, J=6.6,6 \mathrm{H}, 2 \mathrm{CH}_{3}\right), 2.43(\mathrm{~s}, 3 \mathrm{H}$, $\left.\mathrm{COCH}_{3}\right), 3.66\left(\mathrm{~s}, 2 \mathrm{H}, \mathrm{CH}_{2}\right), 4.47$ - $4.56(\mathrm{~m}, 1 \mathrm{H}, \mathrm{NCH})$; Anal. Calcd for $\mathrm{C}_{9} \mathrm{H}_{13} \mathrm{NO}_{3}$ : C, 59.00; H, 7.15; N, 7.65. Found: C, 59.35; $\mathrm{H}, 7.10$; N, 7.72.

1-Butyl-3-(1-hydroxyethylidene)pyrrolidine-2,4-dione (4b): white solid; mp $28.6-29.3^{\circ} \mathrm{C}$; yield, $43.2 \%$; IR $\left(\mathrm{KBr}, \mathrm{cm}^{-1}\right)$ v 2961, 2871, 1714, 1630, 1481, 1378, 1247; ${ }^{1} \mathrm{H} \mathrm{NMR}\left(\mathrm{CDCl}_{3}\right.$, $300 \mathrm{MHz}) \delta 0.91\left(\mathrm{t}, J=7.2,3 \mathrm{H}, \mathrm{CH}_{2} \mathrm{CH}_{3}\right), 1.32-1.34(\mathrm{~m}, 2 \mathrm{H}$, $\left.\mathrm{CH}_{2} \mathrm{CH}_{3}\right), 1.52$ - $1.54\left(\mathrm{~m}, 2 \mathrm{H}, \mathrm{CH}_{2} \mathrm{CH}_{2}\right), 2.42\left(\mathrm{~s}, 3 \mathrm{H}, \mathrm{COCH}_{3}\right)$, $3.42\left(\mathrm{t}, J=7.2,2 \mathrm{H}, \mathrm{NCH}_{2} \mathrm{CH}_{2}\right), 3.70\left(\mathrm{~s}, 2 \mathrm{H}, \mathrm{CH}_{2}\right)$; Anal. Calcd for $\mathrm{C}_{10} \mathrm{H}_{15} \mathrm{NO}_{3}$ : C, 60.90; H, 7.67; N, 7.10. Found: C, 60.53; H, 7.60; N, 7.19.

1-tert-Butyl-3-(1-hydroxyethylidene)pymolidine-2,4-dione (4c): white solid; mp $54.4-55.5^{\circ} \mathrm{C}$; yield, $62.1 \%$; IR $\left(\mathrm{KBr}, \mathrm{cm}^{-1}\right)$ $v 2985,1715,1635,1480,1380,1356,1216 ;{ }^{1} \mathrm{H} \mathrm{NMR}\left(\mathrm{CDCl}_{3}\right.$, $300 \mathrm{MHz}) \delta 1.47\left(\mathrm{~s}, 9 \mathrm{H}, 3 \times \mathrm{CH}_{3}\right), 2.42\left(\mathrm{~s}, 3 \mathrm{H}, \mathrm{COCH}_{3}\right), 3.77$ (s, $2 \mathrm{H}, \mathrm{CH}_{2}$ ); Anal. Calcd for $\mathrm{C}_{10} \mathrm{H}_{15} \mathrm{NO}_{3}$ : C, 60.90; H, 7.67; N, 7.10. Found: C, 61.32; H, 7.59; N, 7.15.

1-Cyclohexyl-3-(1-hydroxyethylidene)pyrrolidine-2,4-dione (4d): white solid; mp $106.0-106.8^{\circ} \mathrm{C}$; yield, 51.1\%; IR (KBr, $\mathrm{cm}^{-1}$ ) v 2939, 2859, 1717, 1640, 1472, 1374, 1251; ${ }^{1} \mathrm{H}$ NMR $\left(\mathrm{CDCl}_{3}, 300 \mathrm{MHz}\right) \delta 1.06-1.88\left(\mathrm{~m}, 10 \mathrm{H}, \mathrm{C}_{6} \mathrm{H}_{10}\right), 2.44(\mathrm{~s}, 3 \mathrm{H}$, $\left.\mathrm{COCH}_{3}\right), 3.68$ (s, 2H, NCH 2$), 4.04$ - 4.16 (m, 1H, NCH); Anal. Calcd for $\mathrm{C}_{12} \mathrm{H}_{17} \mathrm{NO}_{3}$ : C, 64.55; H, 7.67; N, 6.27. Found: C, $64.11 ; \mathrm{H}, 7.58 ; \mathrm{N}, 6.37$.

General procedure for the preparation of the title ompounds 6a-t. To the compound $4(1.5 \mathrm{mmol})$ and $O$-substituted hydroxylamine hydrochloride $\mathbf{5}(2.1 \mathrm{mmol})$ in ethanol $(25 \mathrm{~mL})$ was added $2 \% \mathrm{NaOH}(4.4 \mathrm{~mL})$. Then the solution was refluxed and monitored on TLC. After the reaction completed, water $(50 \mathrm{~mL})$ was added, the organic layer was extracted with ethyl acetate, dried over anhydrous $\mathrm{MgSO}_{4}$. The solvent was removed under reduced pressure. Recrystallization from ether afforded the corresponding compounds 6a-t.

(E)-1-Isopropyl-3-(1-(propoxyamino)ethylidene)pyrolidine2,4-dione (6a): colorless crystal; mp 31.0 - $32.4^{\circ} \mathrm{C}$; yield, $86.2 \%$; IR $\left(\mathrm{KBr}, \mathrm{cm}^{-1}\right) v 3156,2971,2878,1680,1633,1577,1453$, $1402,1237,1063 ;{ }^{1} \mathrm{H}$ NMR $\left(\mathrm{CDCl}_{3}, 300 \mathrm{MHz}\right) \delta 0.97(\mathrm{t}, J=$ $\left.6.9 \mathrm{~Hz}, 3 \mathrm{H}, \mathrm{CH}_{2} \mathrm{CH}_{3}\right), 1.13\left(\mathrm{~d}, J=6.3 \mathrm{~Hz}, 6 \mathrm{H}, \mathrm{CH}\left(\mathrm{CH}_{3}\right)_{2}\right)$, $1.69-1.76\left(\mathrm{~m}, 2 \mathrm{H}, \mathrm{OCH}_{2} \mathrm{CH}_{2}\right), 2.54\left(\mathrm{~s}, 3 \mathrm{H}, \mathrm{CH}_{3} \mathrm{C}=\right), 3.63(\mathrm{~s}$, $\left.2 \mathrm{H}, \mathrm{NCH}_{2}\right), 3.92\left(\mathrm{t}, J=6.7 \mathrm{~Hz}, 2 \mathrm{H}, \mathrm{OCH}_{2}\right), 4.48-4.51(\mathrm{~m}, 1 \mathrm{H}$, $\left.\mathrm{CH}\left(\mathrm{CH}_{3}\right)_{2}\right) ; \mathrm{MS} \mathrm{m} / z(\%): 240\left(\mathrm{M}^{+}, 75\right), 225(100), 166(58), 139$ (31), 124(18), 84(25), 56(46); Anal. Calcd for $\mathrm{C}_{12} \mathrm{H}_{20} \mathrm{~N}_{2} \mathrm{O}_{3}$ : C, 59.98; H, 8.39; N, 11.66. Found: C, 60.42; H, 8.30; N, 11.76.

(E)-3-(1-(Isopropoxyamino)ethylidene)-1-isopropylpymolidine-2,4-dione (6b): damask viscous liquid; yield, 91.2\%; IR $\left(\mathrm{KBr}, \mathrm{cm}^{-1}\right)$ v 3156, 2975, 2933, 1681, 1633, 1578, 1454, 1238, 1113,$1063 ;{ }^{1} \mathrm{H} \mathrm{NMR}\left(\mathrm{CDCl}_{3}, 300 \mathrm{MHz}\right) \delta 1.12(\mathrm{~d}, J=6.6 \mathrm{~Hz}$, 
$\left.6 \mathrm{H}, \mathrm{NCH}\left(\mathrm{CH}_{3}\right)_{2}\right), 1.28\left(\mathrm{~d}, J=6.6 \mathrm{~Hz}, 6 \mathrm{H}, \mathrm{OCH}\left(\mathrm{CH}_{3}\right)_{2}\right), 2.52$ $\left(\mathrm{s}, 3 \mathrm{H}, \mathrm{CH}_{3} \mathrm{C}=\right), 3.61\left(\mathrm{~s}, 2 \mathrm{H}, \mathrm{NCH}_{2}\right), 4.10-4.14(\mathrm{~m}, 1 \mathrm{H}, \mathrm{OCH}$ $\left.\left(\mathrm{CH}_{3}\right)_{2}\right), 4.45$ - $4.48\left(\mathrm{~m}, 1 \mathrm{H}, \mathrm{NCH}\left(\mathrm{CH}_{3}\right)_{2}\right) ; \mathrm{MS} \mathrm{m} / z(\%): 240\left(\mathrm{M}^{+}\right.$, 70), 225(42), 183(100), 139(23), 124(18), 70(20), 56(34); Anal. Calcd for $\mathrm{C}_{12} \mathrm{H}_{20} \mathrm{~N}_{2} \mathrm{O}_{3}$ : C, 59.98; $\mathrm{H}, 8.39 ; \mathrm{N}, 11.66$. Found: $\mathrm{C}$, $60.32 ; \mathrm{H}, 8.45 ; \mathrm{N}, 11.74$.

(E)-3-(1-(Allyloxyamino)ethylidene)-1-isopropylpyrrolidine-2,4-dione (6c): colorless powder; $\mathrm{mp} 45.3$ - $46.2^{\circ} \mathrm{C}$; yield, $75.4 \%$; IR $\left(\mathrm{KBr}, \mathrm{cm}^{-1}\right)$ v 3102, 2972, 2930, 1680, 1633, 1574, $1452,1400,1238,1127 ;{ }^{1} \mathrm{HNMR}\left(\mathrm{CDCl}_{3}, 300 \mathrm{MHz}\right) \delta 1.12(\mathrm{~d}$, $\left.J=6.6 \mathrm{~Hz}, 6 \mathrm{H}, \mathrm{CH}\left(\mathrm{CH}_{3}\right)_{2}\right), 2.51\left(\mathrm{~s}, 3 \mathrm{H}, \mathrm{CH}_{3} \mathrm{C}=\right), 3.63(\mathrm{~s}, 2 \mathrm{H}$, $\left.\mathrm{NCH}_{2}\right), 4.44-4.50\left(\mathrm{~m}, 3 \mathrm{H}, \mathrm{NCH}\left(\mathrm{CH}_{3}\right)_{2}+\mathrm{OCH}_{2}\right), 5.38$ - 5.44 $\left(\mathrm{m}, 2 \mathrm{H}, \mathrm{CH}=\mathrm{CH}_{2}\right), 5.89-6.02\left(\mathrm{~m}, 1 \mathrm{H}, \mathrm{CH}=\mathrm{CH}_{2}\right) ; \mathrm{MS} \mathrm{m} / z(\%)$ : $238\left(\mathrm{M}^{+}, 100\right), 223(83), 167(98), 139(62), 124(33), 84(31), 56$ (82); Anal. Calcd for $\mathrm{C}_{12} \mathrm{H}_{18} \mathrm{~N}_{2} \mathrm{O}_{3}$ : C, 60.49; H, 7.61; N, 11.76 . Found: C, 60.21; H, 7.53; N, 11.81 .

(E)-3-(1-(Butoxyamino)ethylidene)-1-isopropylpymolidine2,4-dione (6d): colorless powder; mp 48.0 - $48.9{ }^{\circ} \mathrm{C}$; yield, $81.6 \%$; IR $\left(\mathrm{KBr}, \mathrm{cm}^{-1}\right)$ v 3162, 2966, 2874, 1682, 1643, 1577, 1453, 1401, 1238, 1064; ${ }^{1} \mathrm{H} \mathrm{NMR}\left(\mathrm{CDCl}_{3}, 300 \mathrm{MHz}\right) \delta 0.92(\mathrm{t}$, $\left.J=7.3 \mathrm{~Hz}, 3 \mathrm{H}, \mathrm{CH}_{2} \mathrm{CH}_{3}\right), 1.13\left(\mathrm{~d}, J=6.9 \mathrm{~Hz}, 6 \mathrm{H}, \mathrm{CH}\left(\mathrm{CH}_{3}\right)_{2}\right)$, $1.37-1.48\left(\mathrm{~m}, 2 \mathrm{H}, \mathrm{CH}_{2} \mathrm{CH}_{3}\right), 1.62-1.72\left(\mathrm{~m}, 2 \mathrm{H}, \mathrm{OCH}_{2} \mathrm{CH}_{2}\right)$, $2.53\left(\mathrm{~s}, 3 \mathrm{H}, \mathrm{CH}_{3} \mathrm{C}=\right), 3.63\left(\mathrm{~s}, 2 \mathrm{H}, \mathrm{NCH}_{2}\right), 3.92(\mathrm{t}, J=6.7 \mathrm{~Hz}$, $\left.2 \mathrm{H}, \mathrm{OCH}_{2}\right), 4.44$ - $4.52\left(\mathrm{~m}, 1 \mathrm{H}, \mathrm{CH}\left(\mathrm{CH}_{3}\right)_{2}\right)$; $\mathrm{MS} m / z(\%): 254$ $\left(\mathrm{M}^{+}, 78\right), 239(100), 167(44), 149(82), 139(32), 69(30), 57(36)$; Anal. Calcd for $\mathrm{C}_{13} \mathrm{H}_{22} \mathrm{~N}_{2} \mathrm{O}_{3}: \mathrm{C}, 61.39 ; \mathrm{H}, 8.72 ; \mathrm{N}, 11.01$. Found: C, 61.01; H, 8.65; N, 11.09.

(E)-3-(1-(Benzyloxyamino)ethylidene)-1-isopropylpymolidine-2,4-dione (6e): colorless crystal; mp 111.3 - $112.2{ }^{\circ} \mathrm{C}$; yield, $83.5 \%$; IR $\left(\mathrm{KBr}, \mathrm{cm}^{-1}\right) v 3182,3035,2968,2925,1664,1624$, $1575,1454,1398,1320,1176 ;{ }^{1} \mathrm{H} \mathrm{NMR}\left(\mathrm{CDCl}_{3}, 300 \mathrm{MHz}\right) \delta$ $1.13\left(\mathrm{~d}, J=6.3 \mathrm{~Hz}, 6 \mathrm{H}, \mathrm{CH}\left(\mathrm{CH}_{3}\right)_{2}\right), 2.43\left(\mathrm{~s}, 3 \mathrm{H}, \mathrm{CH}_{3} \mathrm{C}=\right), 3.65$ (s, $\left.2 \mathrm{H}, \mathrm{NCH}_{2}\right), 4.43$ - $4.51\left(\mathrm{~m}, 1 \mathrm{H}, \mathrm{CH}\left(\mathrm{CH}_{3}\right)_{2}\right), 4.95(\mathrm{~s}, 2 \mathrm{H}$, $\left.\mathrm{OCH}_{2}\right), 7.39$ (m, 5H, PhH); MS m/z (\%): $288\left(\mathrm{M}^{+}, 17\right), 167(43)$, 106(20), 91(100), 77(33), 56(17); Anal. Calcd for $\mathrm{C}_{16} \mathrm{H}_{20} \mathrm{~N}_{2} \mathrm{O}_{3}$ : C, 66.65; H, 6.99; N, 9.72. Found: C, 66.18; H, 6.85; N, 9.87.

(E)-1-Butyl-3-(1-(propoxyamino)ethylidene)pyrrolidine2,4-dione (6f): damask viscous liquid; yield, 93.5\%; IR ( $\mathrm{KBr}$, $\left.\mathrm{cm}^{-1}\right)$ v 3149, 2962, 2930, 2872, 1682, 1636, 1577, 1457, 1238, $1065 ;{ }^{1} \mathrm{H}$ NMR $\left(\mathrm{CDCl}_{3}, 300 \mathrm{MHz}\right) \delta 0.84-0.95(\mathrm{~m}, 6 \mathrm{H}, 2 \times$ $\left.\mathrm{CH}_{2} \mathrm{CH}_{3}\right), 1.22-1.69\left(\mathrm{~m}, 6 \mathrm{H}, 3 \times \mathrm{CH}_{2}\right), 2.49\left(\mathrm{~s}, 3 \mathrm{H}, \mathrm{CH}_{3} \mathrm{C}=\right)$, 3.30 (t, $\left.J=6.6 \mathrm{~Hz}, 2 \mathrm{H}, \mathrm{NCH}_{2} \mathrm{CH}_{2}\right), 3.57$ (s, $\left.2 \mathrm{H}, \mathrm{NCH}_{2}\right), 3.80$ $\left(\mathrm{t}, J=6.3 \mathrm{~Hz}, 2 \mathrm{H}, \mathrm{OCH}_{2}\right) ; \mathrm{MS} m / z(\%): 254\left(\mathrm{M}^{+}, 57\right), 211(54)$, 169(100), 153(25), 112(26), 69(10), 57(9); Anal. Calcd for $\mathrm{C}_{13} \mathrm{H}_{22} \mathrm{~N}_{2} \mathrm{O}_{3}$ : C, 61.39; H, 8.72; N, 11.01. Found: $\mathrm{C}, 61.83 ; \mathrm{H}$, $8.86 ; \mathrm{N}, 10.92$.

(E)-1-Butyl-3-(1-(isopropoxyamino)ethylidene)pymolidine2,4-dione (6g): damask viscous liquid; yield, $84.6 \%$; IR ( $\mathrm{KBr}$, $\left.\mathrm{cm}^{-1}\right) v 3162,2963,2930,1680,1635,1578,1458,1375,1239$, 1112; ${ }^{1} \mathrm{H}$ NMR $\left(\mathrm{CDCl}_{3}, 300 \mathrm{MHz}\right) \delta 0.90(\mathrm{t}, J=7.2 \mathrm{~Hz}, 3 \mathrm{H}$, $\left.\mathrm{CH}_{2} \mathrm{CH}_{3}\right), 1.23\left(\mathrm{~d}, J=6.0 \mathrm{~Hz}, 6 \mathrm{H}, \mathrm{CH}\left(\mathrm{CH}_{3}\right)_{2}\right), 1.24-1.50(\mathrm{~m}$, $\left.4 \mathrm{H}, 2 \times \mathrm{CH}_{2}\right), 2.47\left(\mathrm{~s}, 3 \mathrm{H}, \mathrm{CH}_{3} \mathrm{C}=\right), 3.30(\mathrm{t}, J=6.3 \mathrm{~Hz}, 2 \mathrm{H}$, $\left.\mathrm{NCH}_{2} \mathrm{CH}_{2}\right), 3.58$ (s, $\left.2 \mathrm{H}, \mathrm{NCH}_{2}\right), 4.04$ - $4.14\left(\mathrm{~m}, 1 \mathrm{H}, \mathrm{OCH}\left(\mathrm{CH}_{3}\right)_{2}\right)$; MS $m / z(\%): 254\left(\mathrm{M}^{+}, 65\right), 211(51), 169(100), 153(34), 112(28)$, 45(21); Anal. Calcd for $\mathrm{C}_{13} \mathrm{H}_{22} \mathrm{~N}_{2} \mathrm{O}_{3}: \mathrm{C}, 61.39 ; \mathrm{H}, 8.72 ; \mathrm{N}$, 11.01. Found: C, 61.02; H, 8.81; N, 10.95.

(E)-3-(1-(Allyloxyamino)ethylidene)-1-butylpyrrolidine2,4-dione (6h): damask viscous liquid; yield, $85.1 \%$; IR (KBr, $\left.\mathrm{cm}^{-1}\right) v 3121,2960,2932,1684,1636,1575,1457,1368,1239$, 1026; ${ }^{1} \mathrm{H} \mathrm{NMR}\left(\mathrm{CDCl}_{3}, 300 \mathrm{MHz}\right) \delta 0.90(\mathrm{t}, J=6.9 \mathrm{~Hz}, 3 \mathrm{H}$, $\left.\mathrm{CH}_{2} \mathrm{CH}_{3}\right), 1.25$ - $1.38\left(\mathrm{~m}, 2 \mathrm{H}, \mathrm{CH}_{2} \mathrm{CH}_{3}\right), 1.46-1.55(\mathrm{~m}, 2 \mathrm{H}$, $\left.\mathrm{NCH}_{2} \mathrm{CH}_{2}\right), 2.52\left(\mathrm{~s}, 3 \mathrm{H}, \mathrm{CH}_{3} \mathrm{C}=\right), 3.34(\mathrm{t}, J=6.6 \mathrm{~Hz}, 2 \mathrm{H}$, $\left.\mathrm{NCH}_{2} \mathrm{CH}_{2}\right), 3.70\left(\mathrm{~s}, 2 \mathrm{H}, \mathrm{NCH}_{2}\right), 4.44\left(\mathrm{~d}, J=6.3 \mathrm{~Hz}, 2 \mathrm{H}, \mathrm{OCH}_{2}\right)$, $5.24-5.45\left(\mathrm{~m}, 2 \mathrm{H}, \mathrm{CH}=\mathrm{CH}_{2}\right), 5.90-6.03\left(\mathrm{~m}, 1 \mathrm{H}, \mathrm{CH}=\mathrm{CH}_{2}\right)$; MS $m / z$ (\%): $252\left(\mathrm{M}^{+}, 50\right), 209(62), 153(100), 149(71), 112(26)$, 69(62), 57(78); Anal. Calcd for $\mathrm{C}_{13} \mathrm{H}_{20} \mathrm{~N}_{2} \mathrm{O}_{3}$ : C, 61.88; H, 7.99; N, 11.10. Found: C, 61.39; H, 7.85; N, 10.97.

(E)-3-(1-(Butoxyamino)ethylidene)-1-butylpymolidine-2,4dione (6i): damask viscous liquid; yield, $89.7 \%$; IR ( $\left.\mathrm{KBr}, \mathrm{cm}^{-1}\right)$ v 3111, 2960, 2931, 1684, 1637, 1577, 1457, 1371, 1268, 1070; ${ }^{1} \mathrm{H} \mathrm{NMR}\left(\mathrm{CDCl}_{3}, 300 \mathrm{MHz}\right) \delta 0.88-0.95\left(\mathrm{~m}, 6 \mathrm{H}, 2 \times \mathrm{CH}_{3}\right)$, $1.26-1.70\left(\mathrm{~m}, 8 \mathrm{H}, 4 \times \mathrm{CH}_{2}\right), 2.51\left(\mathrm{~s}, 3 \mathrm{H}, \mathrm{CH}_{3} \mathrm{C}=\right), 3.32(\mathrm{t}, J=$ $\left.7.2 \mathrm{~Hz}, 2 \mathrm{H}, \mathrm{NCH}_{2} \mathrm{CH}_{2}\right), 3.66\left(\mathrm{~s}, 2 \mathrm{H}, \mathrm{NCH}_{2}\right), 3.91(\mathrm{t}, J=6.7 \mathrm{~Hz}$, $\left.2 \mathrm{H}, \mathrm{OCH}_{2}\right) ; \mathrm{MS} \mathrm{m} / z(\%): 268\left(\mathrm{M}^{+}, 53\right), 225(100), 195(25), 153$ (77), 112(23), 84(22), 57(28); Anal. Calcd for $\mathrm{C}_{14} \mathrm{H}_{24} \mathrm{~N}_{2} \mathrm{O}_{3}$ : C, 62.66; H, 9.01; N, 10.44. Found: C, 62.22; H, 8.90; N, 10.41.

(E)-3-(1-(Benzyloxyamino)ethylidene)-1-butylpymolidine2,4-dione (6j): colorless powder; $\mathrm{mp} 52.9$ - $53.9^{\circ} \mathrm{C}$; yield, $92.4 \%$; IR $\left(\mathrm{KBr}, \mathrm{cm}^{-1}\right) v 3141,3032,2958,2930,1680,1635,1573$, $1455,1367,1239 ;{ }^{1} \mathrm{H}$ NMR $\left(\mathrm{CDCl}_{3}, 300 \mathrm{MHz}\right) \delta 0.90(\mathrm{t}, J=$ $\left.7.2 \mathrm{~Hz}, 3 \mathrm{H}, \mathrm{CH}_{2} \mathrm{CH}_{3}\right), 1.25$ - $1.37\left(\mathrm{~m}, 2 \mathrm{H}, \mathrm{CH}_{2} \mathrm{CH}_{3}\right), 1.45$ - 1.55 $\left(\mathrm{m}, 2 \mathrm{H}, \mathrm{NCH}_{2} \mathrm{CH}_{2}\right), 2.43\left(\mathrm{~s}, 3 \mathrm{H}, \mathrm{CH}_{3} \mathrm{C}=\right), 3.32(\mathrm{t}, J=7.2 \mathrm{~Hz}$, $\left.2 \mathrm{H}, \mathrm{NCH}_{2} \mathrm{CH}_{2}\right), 3.68$ (s, $\left.2 \mathrm{H}, \mathrm{NCH}_{2}\right), 4.95\left(\mathrm{~s}, 2 \mathrm{H}, \mathrm{OCH}_{2}\right), 7.39$ (m, 5H, PhH); MS m/z (\%): $302\left(\mathrm{M}^{+}, 12\right), 196(10), 153$ (26), 91(100), 77(24); Anal. Calcd for $\mathrm{C}_{17} \mathrm{H}_{22} \mathrm{~N}_{2} \mathrm{O}_{3}$ : C, 67.53; $\mathrm{H}$, 7.33; N, 9.26. Found: C, 67.24; H, 7.40; N, 9.31.

(E)-1-tert-Butyl-3-(1-(propoxyamino)ethylidene)pyrolidine-2,4-dione (6k): damask viscous liquid; yield, 84.5\%; IR $\left(\mathrm{KBr}, \mathrm{cm}^{-1}\right)$ v 3164, 2969, 2934, 1680, 1630, 1579, 1445, 1366, 1229,$1065 ;{ }^{1} \mathrm{H} \mathrm{NMR}\left(\mathrm{CDCl}_{3}, 300 \mathrm{MHz}\right) \delta 0.93(\mathrm{t}, J=6.9 \mathrm{~Hz}$, $\left.3 \mathrm{H}, \mathrm{CH}_{2} \mathrm{CH}_{3}\right), 1.39\left(\mathrm{~s}, 9 \mathrm{H},\left(\mathrm{CH}_{3}\right)_{3} \mathrm{C}\right), 1.65-1.78\left(\mathrm{~m}, 2 \mathrm{H}, \mathrm{CH}_{2}-\right.$ $\left.\mathrm{CH}_{3}\right), 2.44\left(\mathrm{~s}, 3 \mathrm{H}, \mathrm{CH}_{3} \mathrm{C}=\right), 3.70\left(\mathrm{~s}, 2 \mathrm{H}, \mathrm{NCH}_{2}\right), 3.83$ - 4.87 (t, $\left.J=6.6 \mathrm{~Hz}, 2 \mathrm{H}, \mathrm{OCH}_{2}\right)$; MS $m / z(\%): 254\left(\mathrm{M}^{+}, 92\right), 239(100)$, 180(51), 139(95), 111(40), 83(42), 70(736), 57(48); Anal. Calcd for $\mathrm{C}_{13} \mathrm{H}_{22} \mathrm{~N}_{2} \mathrm{O}_{3}$ : C, 61.39; H, 8.72; N, 11.01. Found: C, 61.64; $\mathrm{H}, 8.60 ; \mathrm{N}, 11.06$.

(E)-1-tert-Butyl-3-(1-(isopropoxyamino)ethylidene)pymolidine-2,4-dione (6l): colorless powder; $\mathrm{mp} 100.4$ - 101.2 ${ }^{\circ} \mathrm{C}$; yield, $85.6 \%$; IR $\left(\mathrm{KBr}, \mathrm{cm}^{-1}\right) v 3100,2975,2939,1656,1622,1570$, 1361, 1322, 1233, 1058; ${ }^{1} \mathrm{H}$ NMR $\left(\mathrm{CDCl}_{3}, 300 \mathrm{MHz}\right) \delta 1.27$ $\left(\mathrm{d}, J=6.3 \mathrm{~Hz}, 6 \mathrm{H}, \mathrm{CH}\left(\mathrm{CH}_{3}\right)_{2}\right), 1.41\left(\mathrm{~s}, 9 \mathrm{H},\left(\mathrm{CH}_{3}\right)_{3} \mathrm{C}\right), 2.50$ (s, $\left.3 \mathrm{H}, \mathrm{CH}_{3} \mathrm{C}=\right), 3.73\left(\mathrm{~s}, 2 \mathrm{H}, \mathrm{NCH}_{2}\right), 4.10-4.17\left(\mathrm{~m}, 1 \mathrm{H}, \mathrm{CH}\left(\mathrm{CH}_{3}\right)_{2}\right)$; MS $m / z(\%): 254\left(\mathrm{M}^{+}, 47\right), 239(56), 181(81), 156(73), 139(100)$, 70(56), 45(95); Anal. Calcd for $\mathrm{C}_{13} \mathrm{H}_{22} \mathrm{~N}_{2} \mathrm{O}_{3}$ : C, 61.39; $\mathrm{H}, 8.72$; N, 11.01. Found: C, 61.48; H, 8.76; N, 10.95.

(E)-3-(1-(Allyloxyamino)ethylidene)-1-tert-butylpymolidine-2,4-dione (6m): colorless crystal; mp 72.1 - $73.2^{\circ} \mathrm{C}$; yield, 81.9\%; IR (KBr, cm $\left.{ }^{-1}\right)$ v 3109, 2974, 2926, 1679, 1630, 1576, $1443,1365,1228,1162,1052 ;{ }^{1} \mathrm{H}$ NMR $\left(\mathrm{CDCl}_{3}, 300 \mathrm{MHz}\right) \delta$ $1.41\left(\mathrm{~s}, 9 \mathrm{H},\left(\mathrm{CH}_{3}\right)_{3} \mathrm{C}\right), 2.47\left(\mathrm{~s}, 3 \mathrm{H}, \mathrm{CH}_{3} \mathrm{C}=\right), 3.76\left(\mathrm{~s}, 2 \mathrm{H}, \mathrm{NCH}_{2}\right)$, $4.43\left(\mathrm{~m}, 1 \mathrm{H}, \mathrm{CH}=\mathrm{CH}_{2}\right), 5.36\left(\mathrm{~d}, 2 \mathrm{H}, J=6.3 \mathrm{~Hz}, \mathrm{OCH}_{2}\right), 5.88$ $6.02\left(\mathrm{~m}, 1 \mathrm{H}, \mathrm{CH}=\mathrm{CH}_{2}\right)$; $\mathrm{MS} \mathrm{m} / z(\%): 252\left(\mathrm{M}^{+}, 44\right), 237(53)$, 181(100), 155(31), 84(43), 70(66), 57(81); Anal. Calcd for $\mathrm{C}_{13} \mathrm{H}_{20} \mathrm{~N}_{2} \mathrm{O}_{3}$ : C, 61.88; H, 7.99; N, 11.10. Found: C, 62.07; H, $8.06 ; \mathrm{N}, 10.97$.

(E)-3-(1-(Butoxyamino)ethylidene)-1-tert-butylpyrrolidine- 
Table 2. Herbicidal and antifungal activities of compounds 6a-t (100 $\mu \mathrm{g} / \mathrm{mL}$, inhibitory rate percent)

\begin{tabular}{|c|c|c|c|c|c|c|}
\hline \multirow{3}{*}{ compd. } & \multicolumn{6}{|c|}{ Ratio(\%) } \\
\hline & \multirow{2}{*}{$\frac{\text { B. campestris }}{\text { Root }}$} & \multicolumn{2}{|c|}{ E. crusgalli } & \multirow{2}{*}{$F$. graminearum } & \multirow{2}{*}{ R. cerealis } & \multirow{2}{*}{ C. orbiculare } \\
\hline & & Root & Stem & & & \\
\hline $6 a$ & $40.8 \pm 4.8$ & $64.1 \pm 2.6$ & $2.6 \pm 3.4$ & $14.8 \pm 1.5$ & $34.1 \pm 2.4$ & $27.5 \pm 1.9$ \\
\hline $6 b$ & $29.4 \pm 1.5$ & $26.0 \pm 3.2$ & $8.2 \pm 1.7$ & $18.6 \pm 0.5$ & $35.8 \pm 1.4$ & $31.7 \pm 2.6$ \\
\hline $6 c$ & $44.2 \pm 3.2$ & $85.5 \pm 1.8$ & $14.3 \pm 2.4$ & $14.8 \pm 1.3$ & $39.2 \pm 2.3$ & $42.1 \pm 0.4$ \\
\hline $6 d$ & $39.0 \pm 3.5$ & $88.1 \pm 1.6$ & 0 & $26.7 \pm 0.2$ & $59.1 \pm 1.8$ & $52.1 \pm 1.5$ \\
\hline $6 e$ & $41.5 \pm 4.9$ & $69.4 \pm 0.9$ & 0 & $29.1 \pm 0.8$ & $46.6 \pm 1.6$ & $50.0 \pm 2.3$ \\
\hline $6 f$ & $61.8 \pm 5.3$ & $41.7 \pm 3.3$ & 0 & $42.9 \pm 1.2$ & $47.4 \pm 0.8$ & $57.5 \pm 2.2$ \\
\hline $6 \mathrm{~g}$ & $60.0 \pm 5.1$ & $36.8 \pm 3.7$ & 0 & $23.8 \pm 2.6$ & $42.7 \pm 2.0$ & $37.1 \pm 1.2$ \\
\hline $6 \mathrm{~h}$ & $52.4 \pm 2.0$ & $75.7 \pm 1.9$ & 0 & $29.1 \pm 0.4$ & $44.0 \pm 1.4$ & $67.5 \pm 0.9$ \\
\hline $6 \mathbf{i}$ & $73.5 \pm 0.5$ & $85.1 \pm 0.9$ & 0 & $31.4 \pm 2.5$ & $55.2 \pm 0.7$ & $55.0 \pm 1.8$ \\
\hline $6 \mathbf{j}$ & $45.4 \pm 2.1$ & $48.9 \pm 3.0$ & 0 & $50.5 \pm 3.6$ & $57.8 \pm 2.8$ & $68.8 \pm 1.5$ \\
\hline $6 \mathrm{k}$ & $46.8 \pm 4.7$ & $47.2 \pm 2.5$ & 0 & $12.3 \pm 0.5$ & $41.1 \pm 4.3$ & $45.0 \pm 2.4$ \\
\hline 61 & $28.9 \pm 4.5$ & $54.8 \pm 1.7$ & 0 & $10.3 \pm 1.3$ & $26.0 \pm 3.1$ & $62.5 \pm 2.6$ \\
\hline $6 \mathrm{~m}$ & $45.7 \pm 3.5$ & $70.5 \pm 1.3$ & 0 & $16.4 \pm 0.5$ & $53.4 \pm 2.6$ & $70.0 \pm 2.7$ \\
\hline $6 n$ & $67.0 \pm 1.3$ & $54.5 \pm 2.8$ & $8.3 \pm 4.1$ & $12.3 \pm 1.9$ & $63.0 \pm 1.2$ & $63.0 \pm 1.0$ \\
\hline 60 & $44.2 \pm 5.6$ & $41.6 \pm 2.4$ & $8.6 \pm 3.6$ & $22.6 \pm 1.5$ & $58.0 \pm 2.4$ & $66.0 \pm 4.2$ \\
\hline $6 p$ & $74.7 \pm 2.0$ & $80.5 \pm 4.2$ & 0 & $19.6 \pm 1.8$ & $42.8 \pm 3.1$ & $44.0 \pm 3.6$ \\
\hline $6 q$ & $54.2 \pm 2.5$ & $66.8 \pm 4.1$ & 0 & $20.4 \pm 5.2$ & $20.9 \pm 0.8$ & $30.7 \pm 2.9$ \\
\hline $6 r$ & $57.3 \pm 3.9$ & $80.7 \pm 1.3$ & 0 & $15.0 \pm 2.7$ & $28.4 \pm 1.6$ & $63.9 \pm 5.1$ \\
\hline $6 s$ & $78.1 \pm 4.0$ & $85.2 \pm 2.5$ & 0 & $31.4 \pm 3.2$ & $48.3 \pm 4.1$ & $52.7 \pm 2.6$ \\
\hline $6 t$ & $37.0 \pm 2.9$ & $16.4 \pm 3.5$ & 0 & $43.6 \pm 2.0$ & $23.9 \pm 1.3$ & $50.2 \pm 1.6$ \\
\hline TeA & $92.3 \pm 2.1$ & $97.8 \pm 0.4$ & $43.1 \pm 2.9$ & $10.0 \pm 1.7$ & $16.1 \pm 3.1$ & $14.5 \pm 3.0$ \\
\hline
\end{tabular}

The values are expressed as means \pm SD of the replicates; $n=3$ for all groups.

2,4-dione (6n): damask viscous liquid; yield, 93.8\%; IR ( $\mathrm{KBr}$, $\left.\mathrm{cm}^{-1}\right)$ v 3127, 2961, 2934, 1680, 1630, 1578, 1444, 1365, 1228,$1069 ;{ }^{1} \mathrm{H} \mathrm{NMR}\left(\mathrm{CDCl}_{3}, 300 \mathrm{MHz}\right) \delta 0.90(\mathrm{t}, J=7.5 \mathrm{~Hz}$, $\left.3 \mathrm{H}, \mathrm{CH}_{2} \mathrm{CH}_{3}\right), 1.40\left(\mathrm{~s}, 9 \mathrm{H},\left(\mathrm{CH}_{3}\right)_{3} \mathrm{C}\right), 1.33-1.40\left(\mathrm{~m}, 2 \mathrm{H}, \mathrm{CH}_{2}-\right.$ $\left.\mathrm{CH}_{3}\right), 1.59-1.66\left(\mathrm{~m}, 2 \mathrm{H}, \mathrm{OCH}_{2} \mathrm{CH}_{2}\right), 2.50\left(\mathrm{~s}, 3 \mathrm{H}, \mathrm{CH}_{3} \mathrm{C}=\right)$, $3.67\left(\mathrm{~s}, 2 \mathrm{H}, \mathrm{NCH}_{2}\right), 3.90\left(\mathrm{t}, J=6.2 \mathrm{~Hz}, 2 \mathrm{H}, \mathrm{OCH}_{2}\right)$; $\mathrm{MS} m / z(\%)$ : $268\left(\mathrm{M}^{+}, 65\right), 253(100), 149(84), 139(73), 70(64), 57(73)$; Anal. Calcd for $\mathrm{C}_{14} \mathrm{H}_{24} \mathrm{~N}_{2} \mathrm{O}_{3}$ : C, 62.66; H, 9.01; N, 10.44. Found: C, 63.07; H, 9.06; N, 10.52 .

(E)-3-(1-(Benzyloxyamino)ethylidene)-1-tert-butylpymolidine-2,4-dione (6o): colorless crystal; mp 98.6 - $99.5^{\circ} \mathrm{C}$; yield, 95.3\%; IR (KBr, cm $\left.{ }^{-1}\right) v 3142,3084,2969,2934,1680,1630$, $1579,1445,1366,1229,1065 ;{ }^{1} \mathrm{H} \mathrm{NMR}\left(\mathrm{CDCl}_{3}, 300 \mathrm{MHz}\right) \delta$ $1.42\left(\mathrm{~s}, 9 \mathrm{H},\left(\mathrm{CH}_{3}\right)_{3} \mathrm{C}\right), 2.40\left(\mathrm{~s}, 3 \mathrm{H}, \mathrm{CH}_{3} \mathrm{C}=\right), 3.76\left(\mathrm{~s}, 2 \mathrm{H}, \mathrm{NCH}_{2}\right)$, 4.96 (s, 2H, $\left.\mathrm{OCH}_{2}\right), 7.38$ (m, 5H, PhH); $\mathrm{MS} \mathrm{m} / z(\%): 302\left(\mathrm{M}^{+}\right.$, 18), 287(10), 181(14), 91(100), 77(11); Anal. Calcd for $\mathrm{C}_{17} \mathrm{H}_{22}-$ $\mathrm{N}_{2} \mathrm{O}_{3}$ : C, 67.53; H, 7.33; N, 9.26. Found: C, 67.93; H, 7.40; N, 9.32.

(E)-1-Cyclohexyl-3-(1-(propoxyamino)ethylidene)pyrrolidine-2,4-dione (6p): colorless powder; $\mathrm{mp} 71.3$ - $72.1^{\circ} \mathrm{C}$; yield, $76.7 \%$; IR $\left(\mathrm{KBr}, \mathrm{cm}^{-1}\right)$ v 3177, 2972, 2855, 1663, 1624, 1574, $1456,1397,1326,1233,1068 ;{ }^{1} \mathrm{H} \mathrm{NMR}\left(\mathrm{CDCl}_{3}, 300 \mathrm{MHz}\right) \delta$ $0.93\left(\mathrm{t}, J=6.9 \mathrm{~Hz}, 3 \mathrm{H}, \mathrm{CH}_{2} \mathrm{CH}_{3}\right), 1.25-1.37\left(\mathrm{~m}, 2 \mathrm{H}, \mathrm{OCH}_{2} \mathrm{CH}_{2}\right)$, $1.61-1.73\left(\mathrm{~m}, 10 \mathrm{H}, 5 \times \mathrm{CH}_{2}\right), 2.50\left(\mathrm{~s}, 3 \mathrm{H}, \mathrm{CH}_{3} \mathrm{C}=\right), 3.60(\mathrm{~s}$, $\left.2 \mathrm{H}, \mathrm{NCH}_{2}\right), 3.87\left(\mathrm{t}, J=6.5 \mathrm{~Hz}, 2 \mathrm{H}, \mathrm{OCH}_{2}\right), 4.00-4.10(\mathrm{~m}, 1 \mathrm{H}$, $\mathrm{NCH})$; MS $m / z$ (\%): $280\left(\mathrm{M}^{+}, 42\right), 221(56), 149(69), 113(33)$, 81(47), 69(54), 45(100); Anal. Calcd for $\mathrm{C}_{15} \mathrm{H}_{24} \mathrm{~N}_{2} \mathrm{O}_{3}$ : C, 64.26; H, 8.63; N, 9.99. Found: C, 64.63; H, 8.50; N, 10.05.
(E)-1-Cyclohexyl-3-(1-(isopropoxyamino)ethylidene)pymolidine-2,4-dione (6q): colorless crystal; $\mathrm{mp} 68.8-69.6^{\circ} \mathrm{C}$; yield, $86.3 \%$; IR $\left(\mathrm{KBr}, \mathrm{cm}^{-1}\right)$ v 3118, 2981, 2930, 1666, 1634, 1575, $1450,1336,1264,1222,1118,1011 ;{ }^{1} \mathrm{H}$ NMR $\left(\mathrm{CDCl}_{3}, 300\right.$ $\mathrm{MHz}) \delta 1.28\left(\mathrm{~d}, J=6.3 \mathrm{~Hz}, 6 \mathrm{H}, \mathrm{CH}\left(\mathrm{CH}_{3}\right)_{2}\right), 1.35-1.80(\mathrm{~m}$, $\left.10 \mathrm{H}, 5 \times \mathrm{CH}_{2}\right), 2.52\left(\mathrm{~s}, 3 \mathrm{H}, \mathrm{CH}_{3} \mathrm{C}=\right), 3.63\left(\mathrm{~s}, 2 \mathrm{H}, \mathrm{NCH}_{2}\right), 4.02-$ $4.12\left(\mathrm{~m}, 2 \mathrm{H}, \mathrm{NCH}+\mathrm{CH}\left(\mathrm{CH}_{3}\right)_{2}\right) ; \mathrm{MS} m / z(\%): 280\left(\mathrm{M}^{+}, 86\right)$, 221(100), 156(48), 139(72), 112(12), 81(25), 55(47); Anal. Calcd for $\mathrm{C}_{15} \mathrm{H}_{24} \mathrm{~N}_{2} \mathrm{O}_{3}$ : C, 64.26; H, 8.63; N, 9.99. Found: $\mathrm{C}$, 64.69; H, 8.69; N, 10.06 .

(E)-3-(1-(Allyloxyamino)ethylidene)-1-cyclohexylpymolidine-2,4-dione (6r): colorless crystal; $\mathrm{mp} 83.0$ - $84.1{ }^{\circ} \mathrm{C}$; yield, $82.4 \%$; IR $\left(\mathrm{KBr}, \mathrm{cm}^{-1}\right) v 3116,2929,2852,1670,1626,1578$, $1448,1392,1318,1179,1071 ;{ }^{1} \mathrm{H} \mathrm{NMR}\left(\mathrm{CDCl}_{3}, 300 \mathrm{MHz}\right) \delta$ $1.29\left(\mathrm{~m}, 10 \mathrm{H}, 5 \times \mathrm{CH}_{2}\right), 2.53\left(\mathrm{~s}, 3 \mathrm{H}, \mathrm{CH}_{3} \mathrm{C}=\right), 3.73(\mathrm{~s}, 2 \mathrm{H}$, $\left.\mathrm{NCH}_{2}\right), 4.03-4.04(\mathrm{~m}, 1 \mathrm{H}, \mathrm{NCH}), 4.03\left(\mathrm{t}, J=6.3 \mathrm{~Hz}, 2 \mathrm{H}, \mathrm{OCH}_{2}\right)$, $5.40-5.46\left(\mathrm{~m}, 2 \mathrm{H}, \mathrm{CH}=\mathrm{CH}_{2}\right), 5.90-6.03\left(\mathrm{~m}, 1 \mathrm{H}, \mathrm{CH}=\mathrm{CH}_{2}\right)$; MS $m / z(\%): 278\left(\mathrm{M}^{+}, 44\right), 222(76), 179(87), 140(10), 124(31)$, 83(55), 55(76); Anal. Calcd for $\mathrm{C}_{15} \mathrm{H}_{22} \mathrm{~N}_{2} \mathrm{O}_{3}$ : C, 64.73; H, 7.97; N, 10.06. Found: C, 65.01; H, 8.04; N, 10.16.

(E)-3-(1-(Butoxyamino)ethylidene)-1-cyclohexylpyrrolidine-2,4-dione (6s): colorless crystal; mp 67.4 - 68.2 ${ }^{\circ} \mathrm{C}$; yield, $89.0 \%$; IR $\left(\mathrm{KBr}, \mathrm{cm}^{-1}\right) v 3154,2932,2855,1666,1626,1577$, $1452,1393,1328,1231,1068 ;{ }^{1} \mathrm{H}$ NMR $\left(\mathrm{CDCl}_{3}, 300 \mathrm{MHz}\right) \delta$ $0.92\left(\mathrm{t}, J=7.5 \mathrm{~Hz}, 3 \mathrm{H}, \mathrm{CH}_{2} \mathrm{CH}_{3}\right), 1.28-1.80\left(\mathrm{~m}, 14 \mathrm{H}, 7 \times \mathrm{CH}_{2}\right)$, $2.53\left(\mathrm{~s}, 3 \mathrm{H}, \mathrm{CH}_{3} \mathrm{C}=\right), 3.65\left(\mathrm{~s}, 2 \mathrm{H}, \mathrm{NCH}_{2}\right), 3.92(\mathrm{t}, J=6.0 \mathrm{~Hz}$, $\left.2 \mathrm{H}, \mathrm{OCH}_{2}\right), 4.03$ - 4.07 (m, 1H, NCH); MS m/z (\%): $294\left(\mathrm{M}^{+}\right.$, 68), 221(100), 179(43), 139(68), 112 (26), 83(31), 55(52); Anal. 
Calcd for $\mathrm{C}_{16} \mathrm{H}_{26} \mathrm{~N}_{2} \mathrm{O}_{3}$ : C, 65.28; H, 8.90; N, 9.52. Found: C, $64.91 ; \mathrm{H}, 8.84 ; \mathrm{N}, 9.46$.

(E)-3-(1-(Benzyloxyamino)ethylidene)-1-cyclohexylpymolidine-2,4-dione (6t): colorless crystal; $\mathrm{mp} 121.4$ - $122.5^{\circ} \mathrm{C}$; yield, $90.6 \%$; IR $\left(\mathrm{KBr}, \mathrm{cm}^{-1}\right) v 3172,3036,2927,2853,1667,1620$, 1574, 1449, 1396, 1261, 1228, 1059; ${ }^{1} \mathrm{H}$ NMR $\left(\mathrm{CDCl}_{3}, 300\right.$ $\mathrm{MHz}) \delta 1.23-1.80\left(\mathrm{~m}, 10 \mathrm{H}, 5 \times \mathrm{CH}_{2}\right), 2.42\left(\mathrm{~s}, 3 \mathrm{H}, \mathrm{CH}_{3} \mathrm{C}=\right), 3.64$ (s, $\left.2 \mathrm{H}, \mathrm{NCH}_{2}\right), 4.00$ - 4.05 (m, 1H, NCH), 4.94 (s, 2H, $\mathrm{OCH}_{2}$ ), 7.38 (m, 5H, $\mathrm{PhH}) ; \mathrm{MS} \mathrm{m} / z$ (\%): 328 (M , 13), 222(51), 179(86), 140(81), 91(95), 77(100), 51(46); Anal. Calcd for $\mathrm{C}_{19} \mathrm{H}_{24} \mathrm{~N}_{2} \mathrm{O}_{3}$ : C, 69.49; H, 7.37; N, 8.53. Found: C, 69.05; H, 7.41; N, 8.42.

\section{Biological Evaluation}

The title compounds were evaluated for their herbicidal activities in Petri plates against Echinochloa crusgalli and Brassica campestris according to a modified method. ${ }^{22}$ The results as shown in Table 2 indicated that all the compounds displayed obvious inhibition activities against the roots of two tested plants at the concentration of $100 \mu \mathrm{g} / \mathrm{mL}$. The inhibitory rates of the compounds $6 \mathbf{i}, \mathbf{6} \mathbf{p}$ and $\mathbf{6 s}$ against the root of $B$. campestris exceeded $70 \%$, and the inhibitory rate of the compounds $\mathbf{6 c}, \mathbf{6 d}$, 6i, 6p, 6rand $\mathbf{6 s}$ against the root of E. crusgalli were over $80 \%$.

In addition, the fungicidal activities of the title compounds in vitro against Fusarium graminearum, Rhizoctonia cerealis and Colletotrichum orbiculare were tested by using a mycelia growth inhibition technique according to the literature. ${ }^{23}$ As Table 2 shown, almost all the compounds exhibited higher fungicidal activities than TeA at the concentration of $100 \mu \mathrm{g} / \mathrm{mL}$. Thereinto, the compound $\mathbf{6 j}$ presented best fungicidal activities with inhibition ratios of more than $50 \%$ against all the tested pathogenic fungi.

Comparing the title compounds containing alkyl or cycloalkyl at 1-position with the similar kind of oxime ether compounds containing substituted phenyl or hydrogen at 1-position, the former presented better inhibiting effect against the weed root and nearly same bioactivity against fungi, but almost lost their inhibition activity against the weed stem. ${ }^{13}$ It is interesting that another pyrrolidine-2,4-dione derivatives with schiff base group at 3-position also exhibited remarkably herbicidal activities and fungicidal activities simultaneity. ${ }^{24}$ It indicated that it is feasible and promising to further modify the structure against 3-position of pyrrolidine-2,4-dione to design and screen new pesticides.

\section{Conclusions}

In summary, a series of novel tetramic acid derivatives containing oxime ether group at 3-position were designed and synthesized. All these compounds were confirmed by IR, MS, ${ }^{1} \mathrm{H}$ NMR spectra and elemental analysis. Especially X-ray single crystal structure diffraction to the compound $6 \mathbf{r}$ made sure the pyrrolidine-2,4-dione moiety and $E$ configuration of the title compounds. According to the preliminary bioassays most of the title compounds exhibited noticeable herbicidal activities especially against the root of E. crusgalli and higher fungicidal activities than the leading compound TeA. Further studies on structural modification and the relationship between structures and biological activities are currently underway.

Acknowledgments. This work was supported by the National High Technology Research and Development Program of China (2006AA10A214), and the Fundamental Research Funds for the Central Universities of China (KYZ200918). We are grateful to Yuan, L. M. for the single crystal data collection. and Lu, A. M. for MS analysis.

Supplementary Material. Crystallographic data for the structure analysis of the compound 6r have been deposited with the Cambridge Crystallographic Data Center, CCDC No. 753455. Copies of these information may be obtained free of charge from The Director, CCDC, 12 Union Road, Cambridge, CB2 1EZ, UK (Fax: +44-1223-336-033; E-mail:deposit@ccdc.cam.ac.uk or URL: http:// www.ccdc.cam.ac.uk).

\section{References}

1. Pogocki, D.; Ruman, T.; Danilczuk, M.; Danilczuk, M.; Celuch, M.; Wałajtys-Rode, E. Eur. J. Pharm. 2007, 563, 18.

2. Nakai, Y.; Tepp, W. H.; Dickerson, T. J.; Johnson, E. A.; Janda, K. D. Bioorg. Med. Chem. 2009, 17, 1152.

3. Vayias, B. J.; Athanassiou, C. G.; Buchelos, C. Th. Crop Protection 2006, 25, 766 .

4. Varma, J.; Dubey, N. K. Intern. J. Food Microbio. 2001, 68, 207.

5. Schobert, R.; Schlenk, A. Bioorg. Med. Chem. 2008, 16, 4203.

6. Dixon, D. J.; Ley, S. V.; Gracza, T.; Szolcsanyi, P. J. Chem. Soc., Perkin Trans. 1 1999, 839.

7. Aoki, S.; Higuchi, K.; Ye, Y.; Satari, R.; Kobayashi, M. Tetrahedron 2000, 56, 1833.

8. Li, J. Y.; Strobel, G.; Harper, J.; Lobkovsky, E.; Clardy, J. Org. Lett. 2000, 2, 767.

9. Chen, S. G.; Yin, C. Y.; Dai, X. B.; Qiang, S.; Xu, X. M. Biochim. Biophys. Acta 2008, 62, 279.

10. Gallardo, G. L.; Peña, N. I.; Chacana, P.; Terzolo, H. R.; Cabrera, G. M. World J. Microb. Biotechnol. 2004, 20, 609.

11. Antony, M.; Gupta, K. P.; Janardanan, K. K.; Mehrotra, N. K. Cancer Lett. 1991, 61, 21.

12. Zhu, Y. Q.; Zou, X. M.; Hu, F. Z.; Yao, C. S.; Liu, B.; Yang, H. Z. J. Agric. Food Chem. 2005, 53, 9566.

13. Zhu, X, J.; Huang, L.; Wang, X. F.; Zhu, Z. Y.; Zheng, X. Q.; Qiang, S.; Yang, C. L. Chi. J. Org. Chem. 2009, 29, 1784.

14. Liu, A. P.; Wang, X. G.; Ou, X. M.; Huang, M. Z.; Duan, X. S.; Wang, Y. L.; Pang, H. L. New Pesticides 2005, 42(4), 3.

15. Song, B. A.; Liu, X. H.; Yang, S.; Hu, D. Y.; Jin, L. H.; Zhang, Y. T. Chi. J. Org. Chem. 2005, 25, 507.

16. Liu, A. P.; Yao, J. R. Chi. J. Pesitic. 2004, 43(5), 196.

17. Yang, C. L.; Qiang, S.; Huang, L.; Zhang, P.; Zhu, Z. Y. CN Pat. $1817859,2006$.

18. Du, Z. T.; Qiu, G. R.; Ma, J. Y.; Wu, T. X.; Pan, X. F. Chem. Reagents 2004, 26(2), 117.

19. Wu, Y. X.; Dai, L. Y. Chi. J. Pestic. 2004, 43(3), 113.

20. Barkley, J. V.; Markopoulos, J.; Markopoulou, O. J. Chem. Soc. Perkin Trans. 2 1994, 1271.

21. Zhu, Z. Y.; Wang, X. F.; Meng, F. G.; Li, Q. B.; Zheng, X. Q.; Qiang, S.; Yang, C. L. J. Heterocyclic Chem. 2010, DOI 10.1002/jhet.452.

22. Luo, Y. P.; Yang, G. F. Bioorg. Med. Chem. 2007, 15, 1716.

23. Chen, X.; Yang, C. L. J. Agric. Food Chem. 2009, 57, 2441.

24. Wang, X. F.; Si, T. F.; Li, Q. B.; Zhu, Z. Y.; Zhu, X. J.; Qiang, S.; Yang, C. L. Arkivoc 2010, ii, 31. 